\title{
Advances in the Diagnosis and Treatment of Painful Diabetic Neuropathy
}

\author{
a report by \\ Mitra Tavakoli, Hassan Fadavi and Rayaz A Malik
}

Division of Cardiovascular Medicine, University of Manchester and Manchester Royal Infirmary DOI:10.17925/EE.2008.04.00.48

Symptoms of painful diabetic neuropathy (PDN) occur in $30-40 \%$ of patients with diabetic neuropathy. ${ }^{1}$ It is most commonly associated with distal symmetrical neuropathy affecting the lower limbs (especially toes and feet), and patients present with burning, stabbing and tingling sensations. PDN is extremely distressing and significantly reduces the quality of life of patients. ${ }^{2}$ Hyperglycaemia is clearly important in the genesis of nerve damage, and recent studies suggest that even minimal perturbations in blood glucose in those with impaired glucose tolerance (IGT) may lead to the development of small nerve fibre damage and neuropathic pain. ${ }^{3}$

The causes and consequences of diabetic neuropathy are complex and not well understood. Several hypotheses have been advocated in an attempt to explain the pathophysiology of diabetic neuropathy and include a combination of increased oxidative stress, advanced glycation, polyol accumulation, decreased nitric oxide and impaired sodium + / potassium ${ }^{+}\left(\mathrm{Na}^{+} / \mathrm{K}^{+}\right)$-adenosine triphosphate (ATPase). ${ }^{4}$ Paradoxically, a lack of treatment for underlying nerve damage has improved our understanding of the natural history of PDN because, although nerve damage may initiate PDN, it is clear that as nerve damage progresses pain may diminish. ${ }^{5}$

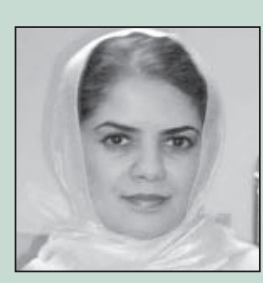

Mitra Tavakoli is a Post-Doctoral Research Fellow in the Cardiovascular Research Group at the University of Manchester. Her research interests focus primarily on the diagnosis of diabetic and other peripheral neuropathies. Dr Tavakoli obtained her PhD from the University of Manchester.

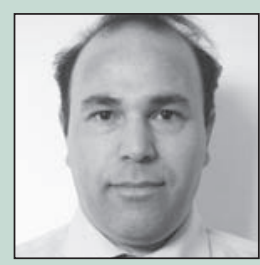

Hassan Fadavi is undertaking his PhD in the Cardiovascular Research Group at the University of Manchester. His main research interests include defining the basis of ethnic differences in the development of diabetic neuropathy and the diagnosis and treatment of diabetic foot ulceration.



Rayaz A Malik is a Professor of Medicine and Consultant Physician in the Division of Cardiovascular Medicine, Manchester Royal Infirmary and University of Manchester. He has clinical responsibility for unselected general acute medicine and a weekly general medical and diabetes outpatient clinic. He is an expert in the pathogenesis, assessment and treatment of diabetic neuropathy. His research is funded by the National Institutes of Health (NIH), the Juvenile Diabetes Research Foundation (JDRF) Diabetes UK and the British Heart Foundation. He is an Associate Editor for Diabetic Medicine, and a reviewer for nine medical journals.

E: rayaz.a.malik@manchester.ac.uk

\section{Diagnosis of Painful Diabetic Neuropathy}

Standard measures of neuropathy such as nerve conduction studies and vibration perception thresholds (VPTs) can be used to detect abnormalities of nerve function, but they focus on large nerve fibres. However, pain is generated and mediated by small $\mathrm{c}$ and $a \delta$ fibres. Thus, it is no surprise that VPT does not differ between diabetic patients with painful and painless neuropathy. ${ }^{6}$ Quantitative sensory testing (QST) including thermal threshold assessment for cold sensation (ad fibres) and warm sensation (c fibres) can assess small fibre dysfunction, but is highly subjective and lacks precision and accuracy, which makes reproducibility difficult. Gore et al. ${ }^{7}$ showed that patients with pain were more likely to have an abnormal cold threshold compared with those without pain; however, the former group also had more severe neuropathy, which was evidenced by a higher VPT and absent reflexes. In general, QST and nerve conduction studies (NCS) cannot distinguish between painful and painless diabetic neuropathy. One of the limitations of QST is the lack of normative data; however, normative data have recently been published for QST ${ }^{8}$ and may allow detection and quantification not only of negative symptoms but also of positive sensory symptoms, such as allodynia, both of which are present in patients with PDN. ${ }^{9}$

The only techniques that allow direct examination of thinly myelinated and unmyelinated nerve fibre damage and repair are sural nerve biopsy with electron microscopy ${ }^{10}$ and skin-punch biopsy, ${ }^{11}$ but both are invasive. Intra-epidermal nerve fibre density can be used to evaluate small fibre involvement in diabetic neuropathy. ${ }^{12}$ However, there is no clear consensus on the role of intra-epidermal nerve fibre (IENF) loss in patients with painful and painless neuropathy. However, some recent studies do suggest that more refined morphometric evaluation of epidermal nerve fibre morphology, such as axonal swellings ${ }^{13}$ and alterations in branching ${ }^{14}$ may be associated with neuropathic pain. A recent study also showed a marked impairment in the cutaneous response to iontophoresed acetylcholine (ACh) in patients with painful neuropathy compared with painless diabetic neuropathy, suggesting that alterations in tissue blood flow may modulate signals generating pain in the periphery. ${ }^{15}$ Recently, we have shown that the novel non-invasive technique of corneal confocal microscopy can detect small fibre neuropathy in diabetic patients by visualising small nerve fibres in Bowman's layer of cornea. ${ }^{15,16}$ Furthermore, it may be more sensitive than IENF density (IENFD) in detecting early damage ${ }^{17}$ and repair after pancreas transplantation. ${ }^{18}$ Thus, this may be an ideal technique to accurately quantify small nerve fibre morphology, especially in a reiterative manner following patients with exacerbations and remissions from PDN. We have recently shown that corneal confocal microscopy (CCM) can be used to demonstrate more advanced small nerve fibre damage in patients with Fabry disease, a hereditary condition with pure small fibre neuropathy (unpublished data). 
Table 1: Treatment Options for Painful Diabetic Neuropathy

\begin{tabular}{|c|c|c|c|c|c|}
\hline Mechanism of Effect & Treatment Method & Drug & Dose per Day (mg) & Side Effects & Comment \\
\hline Optimal glycaemic control & Diet, insulin, exercise & - & - & - & $\begin{array}{l}\text { Contribution of patients and } \\
\text { physician is required }\end{array}$ \\
\hline \multirow[t]{5}{*}{ Pathogenetic treatments } & Pancreas transplantation & - & - & Immunosuppression & $\begin{array}{l}\text { Limited to small centres for } \\
\text { selected patients }\end{array}$ \\
\hline & Alpha-lipoic acid & Alpha-lipoic acid & 600 intravenous & & No data on long-term efficacy \\
\hline & & & $1,200-1,800$ orally & & Only licensed in Germany \\
\hline & Aldose reductase inhibitors & Epalrestat & & & Only licensed in Japan \\
\hline & ACE inhibitors & Trandalopril & & & More studies needed \\
\hline \multirow[t]{13}{*}{$\begin{array}{l}\text { Pharmacological } \\
\text { symptomatic treatment }\end{array}$} & TCAs & Amitriptyline & $20-150$ & +++ & $\begin{array}{l}\text { Sedation and anticholinergic } \\
\text { side effects }\end{array}$ \\
\hline & & Imipramine & $25-150$ & +++ & $\begin{array}{l}\text { Sedation and anticholinergic } \\
\text { side effects }\end{array}$ \\
\hline & SSRIS & Duloxetine & $60-120$ & & Approved by the FDA \\
\hline & & & & & Metabolic side effects \\
\hline & Anticonvulsants & Gabapentin & $900-3,600$ & + & Fewer side effects than TCA \\
\hline & & Lamotrigine & $200-400$ & + & Titration is required \\
\hline & & Carbamazepine & $200-600$ & ++ & Agranulocytosis \\
\hline & & Pregabalin & $300-600$ & & FDA-approved, pedal oedema \\
\hline & Antiarrhythmics & Mexilitene & Up to 900 & +++ & Potential cardiac side effects \\
\hline & Opioids & Tramadol & $50-400$ & ++ & Sedation \\
\hline & & Oxycodone & $40-60$ & +++ & Limited long-term use \\
\hline & Topical agents & Capsaicin cream & Topical & & Denervation \\
\hline & & Topical nitrates & Topical spray & & \\
\hline \multirow[t]{3}{*}{ Physical therapy } & $\begin{array}{l}\text { Electrical spinal- } \\
\text { cord stimulation }\end{array}$ & - & & & Highly invasive \\
\hline & Acupuncture & - & - & & Further research needed \\
\hline & Yoga & - & - & & Further research needed \\
\hline Others & Psychological support & - & - & & Further research needed \\
\hline
\end{tabular}

$A C E=$ angiotensin-converting enzyme; FDA = US Food and Drug Administration; SSRIs = selective serotonin re-uptake inhibitors; TCAs = tricyclic antidepressants; + = severity of side effect.

\section{Assessment of the Severity of Neuropathic Pain}

Assessing the severity of painful symptoms of patients is important, not only for diagnosis but also to assess the benefits of treatments. Many different questionnaires and scores have been developed or adopted to quantify neuropathic pain. The McGill Pain Questionnaire is the most frequently used questionnaire, but it was not originally developed for PDN. Recently, more specific scores have been developed for diabetic painful neuropathy and include the brief pain inventory short form for DPN (BPI-DPN). ${ }^{19}$ The BPI is a patientcompleted numerical rating scale that assesses the severity of pain and its impact on daily functioning on a seven-item pain interference scale. The Neuropathic Pain Questionnaire (NPQ) was developed to provide a general assessment of neuropathic pain and discriminate between neuropathic and non-neuropathic pain.20 The pain diagnostic questionnaire (DN4) is another diagnostic tool that has been shown to compare pain syndromes associated with nervous or somatic lesions. ${ }^{21}$

Follow-up assessment of pain in PDN can be undertaken using either the NPQ or the other recently developed tool, the Neuropathic Pain Symptom Inventory (NPSI), which is a self-questionnaire designed to evaluate different symptoms of neuropathic pain.22 The NPSI includes 10 descriptors that allow for the discrimination and quantification of clinically relevant aspects of neuropathic pain. It has been suggested that this pain questionnaire may be able to characterise subgroups of neuropathic pain patients and verify differential responses to pharmacological or other treatment interventions. Finally, the Neuropathic Pain Scale (NPS) has been specifically designed to monitor effects of therapy on neuropathic pain. ${ }^{23}$

\section{Treatment of Painful Diabetic Neuropathy}

The ideal therapy should prevent or arrest the progressive loss of nerve function and improve symptoms with minimal side effects. However, once pain develops, current treatment options do not address the underlying cause of nerve damage and at best achieve partial alleviation of symptoms, due to significant adverse effects. The treatment of PDN can be focused on three different strategies: treatment based on pathogenetic mechanisms, symptomatic treatments and/or physical and non-pharmacological treatments. Table 1 provides a brief summary of available treatments.

\section{Treatments Based on Pathogenetic Mechanisms}

Intensive glycaemic control is the first priority for both prevention and management of PDN. Recent studies suggest that even minor perturbations in blood glucose in those with IGT may lead to the development of small nerve fibre damage and neuropathic pain. 3,24

\section{Pancreas Transplantation}

The replacement of functioning islet $\beta$-cells by pancreas transplantation has been considered to be the most logical treatment for patients with diabetes to normalise blood glucose and ameliorate long-term complications. Although pancreas transplantation takes approximately five years to prevent progression and 10 years to reverse the lesions of diabetic nephropathy, ${ }^{25}$ a recent study has demonstrated an improvement and/or stabilisation of diabetic retinopathy after a median follow-up of only 17 months. ${ }^{26}$ For diabetic neuropathy, the largest and longest follow-up series to date has shown that pancreas transplantation improved sudomotor function in the hand and foot 
within one year - which was maintained throughout follow-up for 10 years - but had no impact on nerve conduction velocity. ${ }^{27,28}$ Autonomic function did not improve, ${ }^{28}$ and this has been confirmed by another study. ${ }^{29}$ Although in a recent study we have shown that corneal nerves regenerate six months after transplantation, ${ }^{18}$ there is a case report of painful neuropathy worsening after successful transplantation. ${ }^{30}$ However, this treatment option is limited due to a shortage of donated organs, the complications of the procedure and the risks of long-term immunosuppressive therapy. An alternative, less invasive approach is islet cell transplantation, which has recently been shown to improve nerve conduction velocity scores and skin $n$-carboxymethyl-lysine (CML) and receptor for advanced glycation end-product (RAGE) expression, but without evidence of intra-epidermal or sweat gland reinnervation four years after the procedure. ${ }^{31}$

The use of opioids for neuropathic pain remains controversial, as studies have generally been small, yielded equivocal results and have not established the long-term risk-benefit ratio.

\section{Alpha-lipoic acid}

Alpha-lipoic acid (ALA) (thioctic acid) is a powerful antioxidant, and several studies - including the SYDNEY2 trial - have demonstrated an improvement in neuropathic symptoms and deficits. ${ }^{32}$ Results of a meta-analysis provided evidence that treatment with ALA $600 \mathrm{mg} /$ day intravenously over three weeks is safe and significantly improves both positive neuropathic symptoms and neuropathic deficits to a clinically meaningful degree in diabetic patients with symptomatic polyneuropathy. ${ }^{33}$ The only disadvantage of ALA is that it must be given intravenously to achieve maximum benefit. ${ }^{32}$ Of relevance, a recent analysis of two large placebo-controlled clinical intervention trials that included ALA over four years (Nathan 1, Viatris) and a protein kinase $C-\beta$ inhibitor over one year, which had failed to establish efficacy for each compound, showed that most of the endpoints, which included electrophysiology and QSTs, failed to show monotonic worsening in the placebo arm. ${ }^{34}$

\section{Aldose Reductase Inhibitors}

Aldose reductase inhibitors block the enzyme aldose reductase, which has a role in the metabolism of blood glucose via the polyol pathway and may reduce the risk of diabetic neuropathy. Epalrestat is the only aldose reductase inhibitor that has been licensed in Japan. ${ }^{35} \mathrm{~A}$ recent three-year study showed that epalrestat was effective in slowing down the development of neuropathy as measured by changes in nerve conduction compared with controls; however, there was no significant difference in pain between the treated and untreated groups. ${ }^{36}$

\section{Angiotensin-converting Enzyme Inhibitors}

Angiotensin-converting enzyme (ACE) inhibitors have some protective effect against microvascular complications in diabetes, including neuropathy. ${ }^{37}$ In a placebo-controlled study, the ACE inhibitor trandalopril showed an improvement in electrophysiology over 12 months in normotensive patients with mild diabetic neuropathy, but had no impact on QST or painful symptoms. ${ }^{38}$

\section{Symptomatic Treatments}

\section{Pharmacological}

The standard treatment strategy is often first-line tricyclic antidepressants, second-line anticonvulsants and third-line opioid-related treatment. However, only two agents are US Food and Drug Administration (FDA)-approved: duloxetine ${ }^{39}$ and pregabalin. ${ }^{40}$

\section{Antidepressants}

Tricyclic antidepressants (TCAs) have traditionally been first-line therapy for PDN; however, anticholinergic side effects and sedation limit their use, especially in elderly patients. Selective serotonin re-uptake inhibitors (SSRIs) have fewer side effects than TCAs. Most studies with TCAs have enrolled a small number of patients, with still fewer completing the treatment regimens. The most extensively prescribed antidepressant for PDN is amitriptyline, but fewer than 150 patients with PDN have been studied in controlled trials. ${ }^{41,42}$ The major problem remains the frequency of predictable side effects, which include drowsiness and lethargy, and the anticholinergic side effects, particularly dry mouth and postural hypotension. There were also significant problems in defining patients with PDN and the outcome measures employed in each of these trials, thus comparisons cannot be made between studies to determine which drug may have been most beneficial. Duloxetine is the only drug from this class of medications that has received FDA approval for PDN treatment. ${ }^{39}$ Furthermore, a recent UK-based economic model suggests that second-line use of duloxetine is a beneficial and cost-effective treatment strategy for diabetic peripheral neuropathic pain. ${ }^{14}$

\section{Anticonvulsants}

Anticonvulsants have been used in the management of neuropathic pain for many years; however, a recent analysis has found limited evidence for efficacy with this class of drugs in PDN. ${ }^{14}$ Gabapentin is most commonly prescribed, although pregabalin, a higher potency and more effective analogue of gabapentin, is the only other agent apart from duloxetine to have received FDA approval for the treatment of PDN.

\section{Antiarrhythmics}

Mexilitine is a class $1 \mathrm{~B}$ agent that is a structural analogue of lignocaine, but, unlike lidocaine, can be given orally. The results of the Mexiletine Study Group showed that mexiletine at a dosage of $675 \mathrm{mg}$ daily reduced PDN, and the effect of this drug appears to have a rapid onset. ${ }^{43}$ At low doses the risk of electrocardiographic (ECG) side effects is low; however, regular ECG monitoring is necessary and long-term use cannot be recommended. ${ }^{12}$

\section{Opioid-related Treatment}

The use of opioids for neuropathic pain remains controversial, as studies have generally been small, yielded equivocal results and have not established the long-term risk-benefit ratio. ${ }^{44}$ Short-term studies provide only equivocal evidence regarding the efficacy of opioids in reducing the intensity of neuropathic pain, whereas intermediate-term studies demonstrate significant efficacy of opioids over placebo, which is likely to be clinically important. Reported adverse events of opioids, such as sedation and constipation, are common but not life-threatening. Further randomised controlled trials are needed to establish long-term efficacy, safety (including addiction potential) and effects on quality of life. ${ }^{45}$ 


\section{Topical Agents}

\section{Capsaicin}

This is an alkaloid found in chilli peppers and has been shown to be effective in PDN. However, the major clinical concern with this medication is that topical capsaicin has been shown to produce complete or nearly complete denervation of the epidermis in both control subjects and diabetic patients, with a significant reduction in regeneration in the latter. ${ }^{46}$

\section{Topical Nitrates}

A double-blind, placebo-controlled, cross-over study with isosorbide dinitrate spray (a nitric oxide donor with local vasodilating properties) showed a significant reduction in overall pain and burning discomfort over four weeks compared with controls, although the lack of a placebo effect was not consistent with most other studies in PDN. ${ }^{47}$

\section{Physical and Non-pharmacological Treatments}

For patients with more refractory PDN or who suffer from significant side effects of pharmacotherapy, there are some non-pharmacological options. Use of acupuncture is supported by some studies; 48,49 however, a placebo-controlled study has not been performed. Frequencymodulated electromagnetic neural stimulation ${ }^{50}$ has been reported to provide long-term relief for some patients with painful symptoms.

\section{Conclusion}

PDN is a common, difficult to manage, distressing and disabling complication of diabetes. Apart from improving glycaemic control, we have no licensed treatment for diabetic neuropathy. Although we have many approaches to the treatment of PDN, achieving $>50 \%$ relief is rare and side effects limit dose titration. Currently, there are only two FDA-approved medications for PDN. Thus, prevention, early diagnosis and improvement in the understanding of the pathogenesis of pain in diabetic neuropathy may lead to new, more targeted treatments with greater efficacy and fewer side effects.
1. Mojaddidi M, Quattrini C, Tavakoli M, Malik RA, Recent developments in the assessment of efficacy in clinical trials of diabetic neuropathy, Curr Diab Rep, 2005;5:417-22.

2. Zelman DC, Brandenburg NA, Gore M, Sleep impairment in patients with painful diabetic peripheral neuropathy, Clin J Pain, 2006;22:681-5.

3. Smith $A G$, Singleton JR, Impaired glucose tolerance and neuropathy, Neurologist, 2008:14:23-9.

4. Tavakoli M, Mojaddidi M, Fadavi H, Malik RA, Pathophysiology of painful diabetic neuropathy and its treatment, Curr Pain Headache Rep, 2008;12:192-7.

5. Corbett $C F$, Practical management of patients with painful diabetic neuropathy, Diabetes Educ, 2005;31:523-4, 526-8, 530 passim.

6. Sorensen L, Molyneaux L, Yue DK, The relationship among pain, sensory loss, and small nerve fibers in diabetes, Diabetes Care, 2006;29:883-7.

7. Gore M, Dukes E, Rowbotham DJ, et al., Clinical characteristics and pain management among patients with painful peripheral neuropathic disorders in general practice settings, Eur J Pain, 2007;11:652-64.

8. Calcutt NA, Backonja MM, Pathogenesis of pain in peripheral diabetic neuropathy, Curr Diab Rep, 2007;7:429-34.

9. Kelly KG, Cook T, Backonja MM, Pain ratings at the thresholds are necessary for interpretation of quantitative sensory testing, Muscle Nerve, 2005;32:179-84.

10. Malik RA, Tesfaye $S$, Newrick $P G$, et al., Sural nerve pathology in diabetic patients with minimal but progressive neuropathy, Diabetologia, 2005:48:578-85.

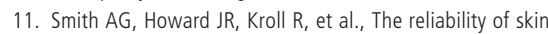
biopsy with measurement of intraepidermal nerve fiber density, J Neurol Sci, 2005;228:65-9.

12. Boulton AJ, Treatment of symptomatic diabetic neuropathy, Diabetes Metab Res Rev, 2003;19(Suppl. 1)S16-21.

13. Lauria $G$, Morbin M, Lombardi $R$, et al., Axonal swellings predict the degeneration of epidermal nerve fibers in painful neuropathies, Neurology, 2003;61:631-6.

14. Beard SM, McCrink L, Le TK, et al., Cost effectiveness of duloxetine in the treatment of diabetic peripheral neuropathic pain in the UK, Curr Med Res Opin, 2008:24:385-99.

15. Quattrini C, Tavakoli M, Jeziorska M, et al., Surrogate markers of small fiber damage in human diabetic neuropathy, Diabetes, 2007:56:2148-54.

16. Hossain P, Sachdev A, Malik RA, Early detection of diabetic peripheral neuropathy with corneal confocal microscopy, Lancet, 2005:366:1340-43.

17. Chalk C, Benstead TJ, Moore F, Aldose reductase inhibitors for the treatment of diabetic polyneuropathy, Cochrane Database Syst Rev, 2007;CD004572.

18. Mehra S, Tavakoli M, Kallinikos PA, et al., Corneal confocal microscopy detects early nerve regeneration after pancreas transplantation in patients with type 1 diabetes, Diabetes Care, 2007;30:2608-12.

19. Zelman DC, Gore M, Dukes $E$, et al., Validation of a modified version of the brief pain inventory for painful diabetic peripheral neuropathy, I Pain Symptom Manage, 2005;29:401-10

20. Krause SJ, Backonja MM, Development of a neuropathic pain questionnaire, Clin J Pain, 2003;19:306-14.

21. Bouhassira D, Attal N, Alchaar $\mathrm{H}$, et al., Comparison of pain syndromes associated with nervous or somatic lesions and development of a new neuropathic pain diagnostic questionnaire (DN4), Pain, 2005;114:29-36.

22. Bouhassira D, Attal N, Fermanian J, et al., Development and validation of the Neuropathic Pain Symptom Inventory, Pain 2004;108:248-57.

23. Galer BS, Jensen MP, Development and preliminary validation of a pain measure specific to neuropathic pain: the Neuropathic Pain Scale, Neurology, 1997;48:332-8

24. Singleton J, Bixby B, Feldman EL, et al., Diet and exercise counselling alone does not prevent long term neuropathy progression in IGT neuropathy, Neurology, 2007;68:A410.

25. Fioretto $P$, Steffes MW, Sutherland DE, et al., Reversal of lesions of diabetic nephropathy after pancreas transplantation, N Engl J Med, 1998:339:69-75.

26. Giannarelli R, Coppelli A, Sartini M, et al., Effects of pancreas-kidney transplantation on diabetic retinopathy, Transpl Int, 2005;18:619-22.

27. Kennedy WR, Navarro X, Goetz FC, et al., Effects of pancreatic transplantation on diabetic neuropathy, $N$ Engl J Med, 1990; 322:1031-7.

28. Navarro $X$, Sutherland DE, Kennedy WR, Long-term effects of pancreatic transplantation on diabetic neuropathy, Ann Neurol, 1997;42:727-36.

29. Boucek P, Saudek F, Adamec M, et al., Spectral analysis of heart rate variation following simultaneous pancreas and kidney transplantation, Transplant Proc, 2003;35:1494-8.

30. Norden G, Olausson M, Andersen O, A case of painful progressive peripheral neuropathy after successful pancreas transplantation, J Diabet Complications, 1991;5:249-51.

31. Del Carro U, Fiorina P, Amadio S, et al., Evaluation of polyneuropathy markers in type 1 diabetic kidney transplant patients and effects of islet transplantation: neurophysiological and skin biopsy longitudinal analysis, Diabetes Care, 2007;30: 3063-9.

32. Ziegler $D$, Ametov A, Barinov A, et al., Oral treatment with alpha-lipoic acid improves symptomatic diabetic polyneuropathy: the SYDNEY 2 trial, Diabetes Care, 2006;29 2365-70.

33. Ziegler $D$, Nowak $H$, Kempler $P$, et al., Treatment of symptomatic diabetic polyneuropathy with the antioxidant alpha-lipoic acid: a meta-analysis, Diabet Med, 2004;21: 114-21.

34. Dyck P, Norell JE, Tritschler $\mathrm{H}$, et al., Challenges in design of multicenter trials: end points assessed longitudinally for change and monotonicity, Diabetes Care, 2007;30:2619-25.

35. Hotta N, Toyota T, Matsuoka K, et al., Clinical efficacy of fidarestat, a novel aldose reductase inhibitor, for diabetic peripheral neuropathy: a 52-week multicenter placebocontrolled double-blind parallel group study, Diabetes Care, 2001;24:1776-82.

36. Hotta N, Akanuma Y, Kawamori R, et al., Long-term clinical effects of epalrestat, an aldose reductase inhibitor, on diabetic peripheral neuropathy: the 3-year, multicenter, comparative Aldose Reductase Inhibitor-Diabetes Complications Trial, Diabetes Care, 2006;29:1538-44.

37. Fried $L F$, Forrest $K Y$, Ellis $D$, et al., Lipid modulation in insulin-dependent diabetes mellitus: effect on microvascular outcomes, I Diabetes Complications, 2001;15:113-19.

38. Malik RA, Williamson S, Abbott $C$, et al., Effect of angiotensin-converting-enzyme (ACE) inhibitor trandolapril on human diabetic neuropathy: randomised double-blind controlled trial, Lancet, 1998;352:1978-81.

39. Wernicke JF, Pritchett YL, D'Souza DN, et al., A randomized controlled trial of duloxetine in diabetic peripheral neuropathic pain, Neurology, 2006;67:1411-20.

40. Gilron I, Bailey JM, Tu D, et al., Morphine, gabapentin, or their combination for neuropathic pain, $N$ Engl I Med, 2005:352 1324-34.

41. Max MB, Lynch SA, Muir J, et al., Effects of desipramine, amitriptyline, and fluoxetine on pain in diabetic neuropathy, N Engl J Med, 1992;326:1250-56.

42. Vrethem M, Boivie J, Arnqvist $H$, et al., A comparison a amitriptyline and maprotiline in the treatment of painful polyneuropathy in diabetics and nondiabetics, Clin I Pain, 1997; 13:313-23.

43. Oskarsson $P$, Ljunggren JG, Lins PE, Efficacy and safety of mexiletine in the treatment of painful diabetic neuropathy. The Mexiletine Study Group, Diabetes Care, 1997;20:1594-7.

44. Veves A, Backonja M, Malik RA, Painful Diabetic Neuropathy: Epidemiology, Natural History, early Diagnosis, and Treatment Options, Pain Medicine, 2007, in press.

45. Eisenberg E, McNicol E, Carr DB, Opioids for neuropathic pain, Cochrane Database Syst Rev, 2006;3:CD006146.

46. Polydefkis $M$, Hauer $P$, Sheth $S$, et al., The time course of epidermal nerve fibre regeneration: studies in normal controls and in people with diabetes, with and without neuropathy, Brain, 2004;127:1606-15.

47. Yuen KC, Baker NR, Rayman G, Treatment of chronic painful diabetic neuropathy with isosorbide dinitrate spray: a doubleblind placebo-controlled cross-over study, Diabetes Care, 2002; 25:1699-1703.

48. Head KA, Peripheral neuropathy: pathogenic mechanisms and alternative therapies, Altern Med Rev, 2006;11:294-329.

49. Jiang H, Shi K, Li X, et al., Clinical study on the wrist-ankle acupuncture treatment for 30 cases of diabetic peripheral neuritis, J Tradit Chin Med, 2006;26:8-12.

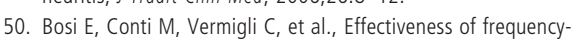
modulated electromagnetic neural stimulation in the treatment of painful diabetic neuropathy, Diabetologia, 2005;48:817-23. 